\title{
SURFACE GRADING USING DEEP LEARNING APPROACH FOR CERAMIC TILES
}

\author{
Dr. Raja V.L, \\ Department of Mechanical Engineering, \\ Muthayammal Engineering College, \\ Namakkal, Salem, India \\ vlraja@rediffmail.com
}

\begin{abstract}
The surface grading of ceramic tiles is essential for ceramic tile industries due to the huge development of infrastructure and essential usage of ceramic tiles. In some industries, surface grading is performed manually. It is a difficult task due to a large number of variations in the surface properties. In this study, a technique for surface grading of ceramic using deep learning is presented. The system uses the VxC Tiles of Surface Grading (TSG) database for performance evaluation. The deep learning based Convolution Neural Network (CNN) is used for the surface grading approach that classifies the tiles into Grade-1 (G1), Grade-2 (G2) and Grade-3 (G3). The system uses seven layers in CNN, which includes convolution, pooling and fully connected layers. Initially, the input tile image is converted Red, Green and Blue (RGB) color channels, and then CNN approach is applied for the classification of tile images. Experimental results show the better classification accuracy of $96.17 \%$ for surface grading of ceramic tiles using a deep learning approach.
\end{abstract}

Keywords: Surface grading, ceramic tiles, VxC-TSG database, convolution neural network

\section{INTRODUCTION}

The surface defect detection from the plain ceramic tiles using image processing is presented in [1]. The acquired input tile images are preprocessed by image enhancement technique and noise removal by low pass filter. Then, the edges are detected. A morphological operation is used to remove unwanted regions. If there is no white pixel in the image, the spot is detected. Ceramic tile images with dot product for corner defect detection are described in [2]. Initially, the input image is cropped in the preprocessing stage. Then, segmentation technique is employed by thresholding technique. The median filtering approach is used to remove noise and then each initial point is identified in each boundary. Feature extraction is made by geometric explanation and conversion between radius and degrees. The decision-making algorithm is used for detection.

Ceramic tile location based on a combination of features is discussed in [3]. Firstly, the gauss pyramid model is used to reduce the search space, by smoothing and raw data are reduced. Geometric features and color features make the feature extraction. The localization of tile images is made by parameter optimization and target discriminating localization method. Automated defect detection in ceramic tiles using image processing is described in [4]. At first, the preprocessing steps such as resizing the image and colour to gray conversion are performed. Morphological operations are performed to remove redundant regions. Then, the good tile classification is made by color variation method.

Surface grading of ceramic tiles based on the feature fusion technique is presented in [5]. A Discrete Wavelet Transform (DWT) decomposes the input tile images. Then the features are extracted by gray level co-occurrence matrix. 
Statistical features like contrast, correlation, homogeneity and energy are also extracted. Then the classification is made by feed-forward neural network classifier. An efficient and simple method for ceramic tile surface defects is presented in [6]. The image preprocessing is made by intensity adjustment, contrast, image acquisition and surface extraction technique. Feature extraction is made by reference tile comparison methods and self-learning methods for the ceramic tile classification.

Colour histogram based colour grading of randomly textured ceramic tiles is discussed in [7]. At first, the histogram of test tile is computed, then the color histogram is compared for the color shade grading. The features like divergence, Bhattacharyya distance, chi-square, correlation coefficient and matusita distance are extracted. The production line is stimulated. Then the color histogram smoothing is made for color grading of ceramic tiles.

The color grading of ceramic tiles using machine vision is presented in [8]. The $k$-means clustering is applied to the input tile images. Then the tiles are graded into two sets by using their inter-class difference. The automatic color grading of ceramic tiles is discussed in [9] based on the combination of color and DWT analysis. DWT is applied to the input tile images. The subband entropy features are computed and then feature selection is applied. Finally, the $k$-nearest neighbor is used for ceramic tile grading.

Marble plate classification based on a neural network is described in [10]. The three types of feature extraction techniques like DWT, discrete cosine transform and texture histograms are used. A Multi Layer Perceptron (MLP) makes the marble classification. Marble surface classification using DWT is presented in [11]. Visual quality parameters are extracted from DWT coefficients and the obtained parameters decides the surface quality of marbles.

A technique for surface grading of ceramic tiles using deep learning approach is presented. The organization of the paper is as follows: The materials and methods for surface grading of ceramic tiles are presented in section 2 . In section 3, experimental results and discussion of the surface grading system are presented. The last section concludes the surface grading system by deep learning approach.

\section{METHODS AND MATERIALS}

In this study, deep learning based surface grading of ceramic tiles is presented. At first, the RGB channels are separated from the input tile images. Then deep learning based CNN approach is employed as feature extraction and also for the classification of tile images. Figure 1 shows the work flow of surface grading of ceramic tiles based on CNN.

\section{A. Separation of RGB color channels}

RGB refers to three additive colors such as Red, Green and Blue. The color pixel of each image with the combination of red, green and blue intensities are stored in each color plane in the pixel location. RGB images are referred as true color images due to their color intensities. RGB images in graphic file format are stored as 24-bit images with 8-bits for each Red, Green and Blue component. 


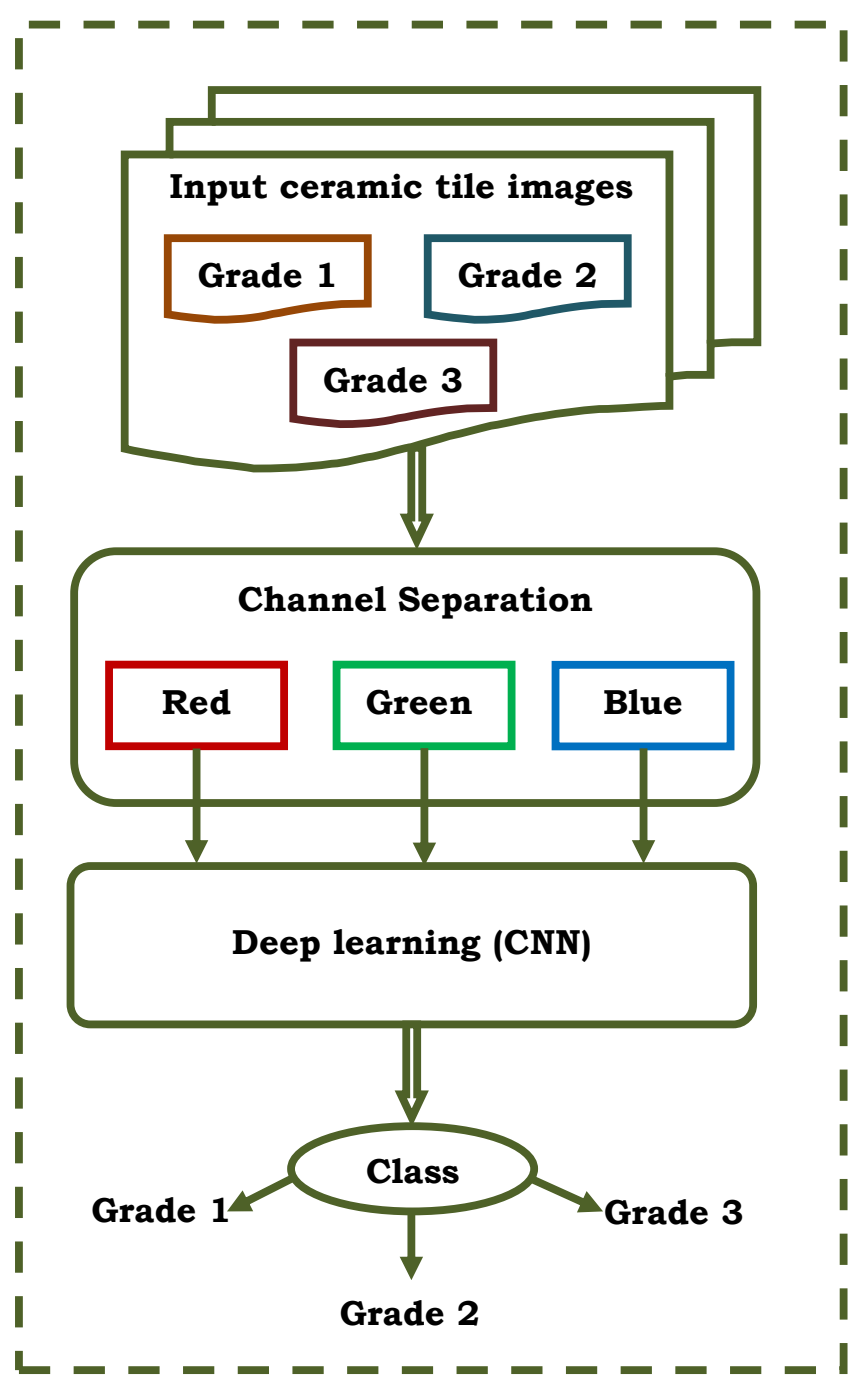

Fig. 1 Surface grading in ceramic tiles using CNN - workflow

RGB color model represents the display of images in electronic systems such as computers, laptops and televisions also used in photography. RGB channels are directly used in image de-blurring [12] and segmentation of lesion in leaf crops [13]. In this study, the input color tile images are separated into three channels at first, and then deep learning based CNN technique is used for the classification.

\section{B. Deep learning based CNN fusion}

CNN is a standard version of MLP. In a fully connected layer MLP is connected to all neurons in the next layer. It causes over fitting data and some measurement and weights may cause loss function. CNN overcomes MLP drawback by using hierarchical pattern in data. It represents vast complex pattern by using simpler and smaller patterns. 
When comparing with other image processing techniques, CNN uses only low pre-processing steps. It is also used in lung abnormalities [14] and electrocardiogram classification [15]. Figure 2 shows the deep learning based CNN architecture for surface grading in ceramic tiles. The output from the CNN approach G1, G2 and G3 represents Grade 1, Grade 2 and Grade 3 respectively.

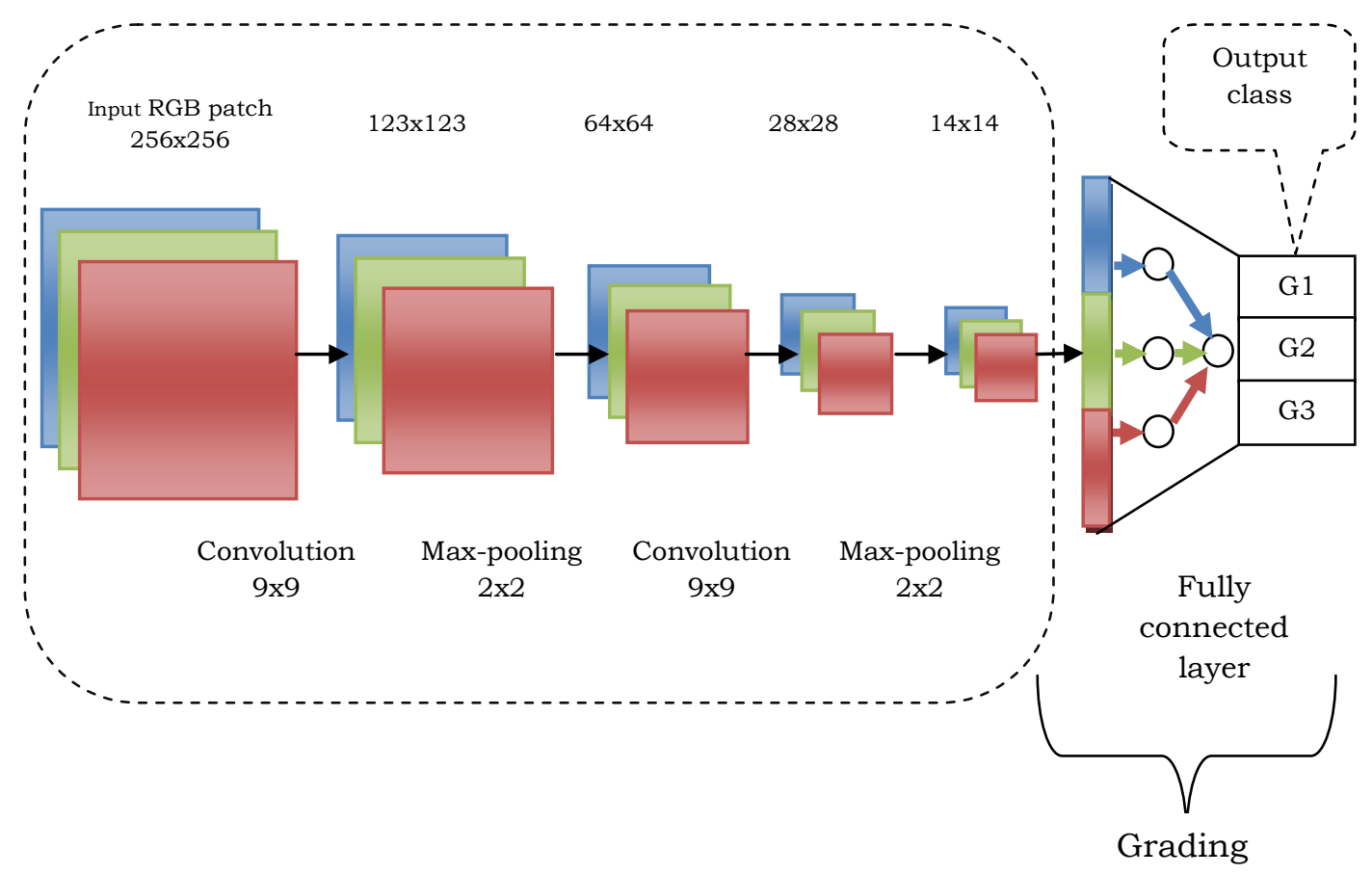

Fig. 2 Deep learning based CNN architecture for surface grading ceramic tiles

In this study, CNN architecture is used for the classification of ceramic tile images. All tile images are resized (128 x 128 pixels) to a standard size and their colour channels are separated. Each colour channel is convoluted with a 9x9 filter and then rectilinear activation function is applied to extract the feature map. Finally, max-pooling with stride 2 is applied that selects the largest value inside the matrix of size $2 \times 2$. This process is applied twice to get the reduced feature map of size $14 \times 14$ for each colour channel. The fully connected layer is used for the classification of ceramic tile images.

\section{RESULTS AND DISCUSSION}

The performance of surface grading system is evaluated by VxC TSG image database [16]. The database images are taken from ceramic industry which consists of 960 digital images with 42 surface grades and 14 different ceramic models. Database consists of three types of ceramic patterns like pseudo random, random and fixed. Table 1 shows the description of VxC TSG database. Figure 3 shows the different surface grades in VxC TSG database like Mediteranea, Antique and Santiago, with G1, G2 and G3. 
TABLE 1 VXC TSG database- description

\begin{tabular}{|c|c|c|c|c|c|}
\hline Name & Tiles/Class & Pattern & Classes & Size (cm) & Aspect \\
\hline Venice & 20 & Pseudorandom & $12,17,18$ & $20 \times 25$ & Marble \\
\hline Vega & 20 & Fixed & $30,31,37$ & $20 \times 25$ & Marble \\
\hline Marfil & 14 & Pseudorandom & $27,32,33$ & $23 \times 33$ & Marble \\
\hline Agata & 16 & Fixed & $13,37,38$ & $33 \times 33$ & Marble \\
\hline Berlin & 24 & Random & $2,3,11$ & $20 \times 20$ & Granite \\
\hline Lima & 24 & Random & $1,4,17$ & $20 \times 20$ & Granite \\
\hline Oslo & 24 & Random & $2,3,7$ & $20 \times 20$ & Granite \\
\hline Somport & 28 & Random & $34,35,38$ & $19 \times 19$ & Stone \\
\hline Santiago & 28 & Random & $22,24,25$ & $19 \times 19$ & Stone \\
\hline Petra & 28 & Random & $7,9,10$ & $16 \times 16$ & Stone \\
\hline Mediterranea & 30 & Random & $1,2,7$ & $20 \times 20$ & Stone \\
\hline
\end{tabular}

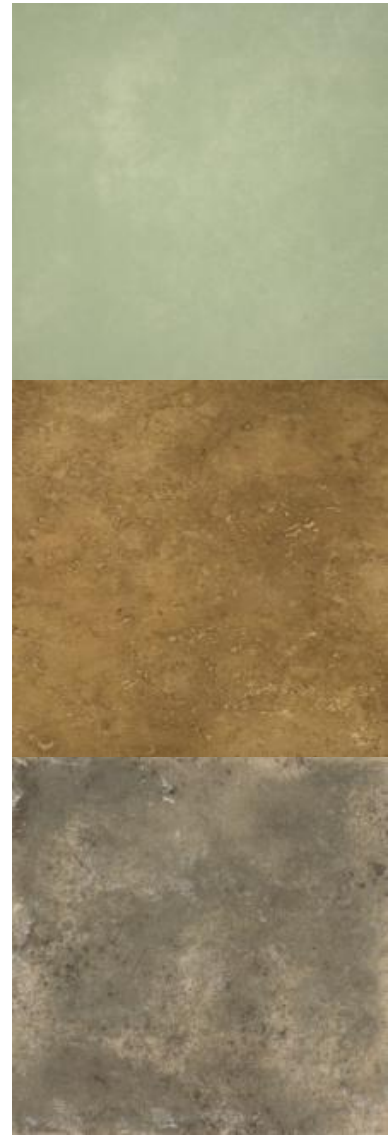

G1

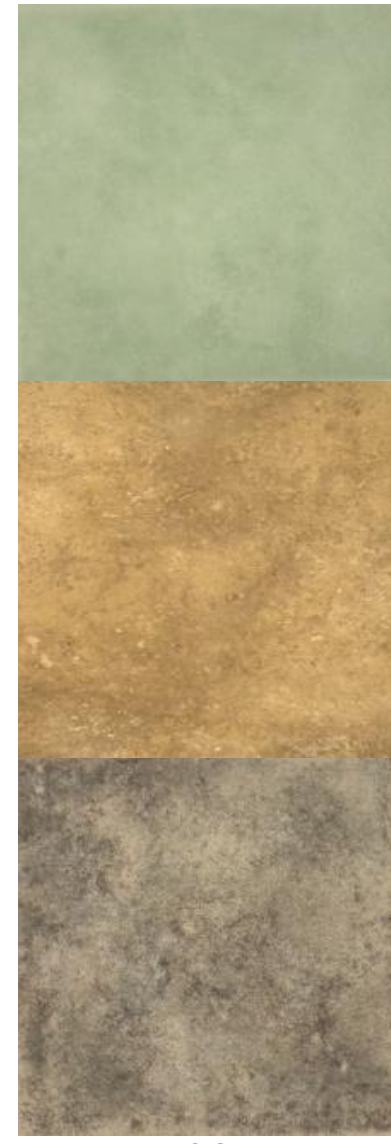

G2

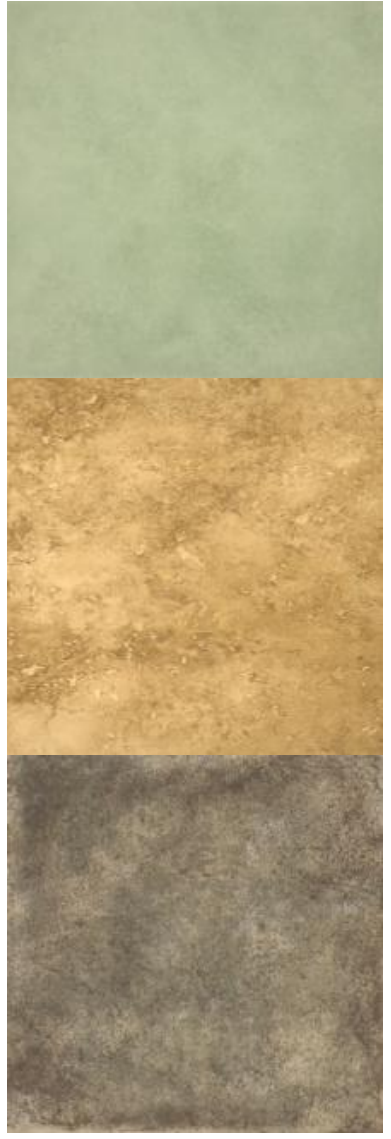

G3

Fig. 3 Sample images of different surface grades in VxC TSG database Mediterranea (top row), Antigue (middle row) and Santiago (bottom row)

The surface grading of ceramic tile images is made by using VxC TSG database. Initially, the input ceramic tile images are converted into RGB channels. These three channels (Red, Green and Blue) given separately for the classification. 
The deep learning based CNN method is used for classification. The fully connected layer in the CNN grades the surface of the given tiles. Table 2 shows the classification accuracy of surface grading in ceramic tiles using CNN.

TABLE 2 Classification accuracy of surface grading in ceramic tiles using CNN

\begin{tabular}{|c|c|c|c|c|}
\hline \multirow{2}{*}{ Database } & \multicolumn{3}{|c|}{ Classification Accuracy (\%) } & \multirow{2}{*}{ Average (\%) } \\
\cline { 2 - 4 } & G1 & G2 & G3 & \\
\hline Venice & 98 & 100 & 99 & 99 \\
\hline Vega & 96 & 98 & 100 & 98 \\
\hline Marfil & 100 & 99 & 96 & 98.3 \\
\hline Agata & 98 & 96 & 94 & 96 \\
\hline Berlin & 100 & 94 & 98 & 97.3 \\
\hline Lima & 99 & 92 & 99 & 96.6 \\
\hline Oslo & 97 & 90 & 100 & 95.6 \\
\hline Somport & 100 & 98 & 98 & 98.6 \\
\hline Santiago & 95 & 100 & 93 & 96 \\
\hline Petra & 93 & 90 & 89 & 90.6 \\
\hline Mediterranea & 91 & 98 & 98 & 95.6 \\
\hline Firenze & 100 & 96 & 96 & 97.3 \\
\hline Campinya & 89 & 93 & 100 & 94 \\
\hline Antique & 93 & 90 & 98 & 93.6 \\
\hline Overall Average (\%) & 96.35 & 95.5 & 97 & $\mathbf{9 6 . 1 7}$ \\
\hline
\end{tabular}

It is observed from Table 2 that the overall classification accuracy of $96.17 \%$ is obtained by using the deep learning based on CNN approach. The average classification accuracy of G1, G2 and G3 tile images are 96.35\%, 95.5\% and $97 \%$. Among the three grades of surfaces in the tile images, G3 tile images have the better classification accuracy comparing with G1 and G2 tile images by using deep learning based CNN approach.

\section{CONCLUSION}

An efficient method for surface grading of ceramic tiles using CNN approach is presented. The input ceramic tile images of Grade 1, 2 and 3 are converted into RGB channels. Then, the red, green and blue channels are separately given to the CNN approach. In this study, CNN with seven layers is used. The first two convolution layers are applied with relu activation function for better classification. Max-pooling layer is applied to two times for better accuracy. Then, $3 \times 3$ matrices with separate $\mathrm{R}, \mathrm{G}$ and $\mathrm{B}$ channels are given to fully connected layer for the classification. A better classification accuracy of $96.17 \%$ is attained for surface grading of ceramic tiles using deep learning approach based CNN. 


\section{REFERENCES}

[1]. A.N. Shire, M.M. Khanapurkar, and R.S. Mundewadikar, "Plain ceramic tiles surface defect detection using image processing", IEEE International Conference on Emerging Trends in Engineering \& Technology, 2011, pp. 215-220.

[2]. F.S. Najafabadi, and H. Pourghassem, "Corner defect detection based on dot product in ceramic tile images", IEEE International Colloquium on Signal Processing and its Applications, 2011, pp. 293-297.

[3]. L. Yang, Q. Li, C. Bai, and J. Li, "A Location Method for Ceramic Tile Based on the Combination Features", IEEE International Conference on Mechanical, Control and Computer Engineering, 2017, pp. 62-66.

[4]. Y.C. Samarawickrama, and C.D. Wickramasinghe, "Matlab based automated surface defect detection system for ceramic tiles using image processing", National Conference on Technology and Management, 2017, pp. 34-39.

[5]. M. Senthilkumar, V. Palanisamy, and J, Jaya, "An Efficient Feature Fusion Technique for Surface Grading of Ceramic Tiles", Middle-East Journal of Scientific Research, Vol. 23, No. 1, 2015, pp. 59-65.

[6]. Z. Hocenski, T. Keser, and A. Baumgartner, "A simple and efficient method for ceramic tile surface defects detection", IEEE International Symposium on Industrial Electronics, 2007, pp. 1606-1611.

[7]. C. Boukouvalas, J. Kittler, R. Marik, and M. Petrou, "Color grading of randomly textured ceramic tiles using color histograms", IEEE Transactions on industrial electronics, Vol. 46, No. 1, 1999, pp. 219-226.

[8]. C. Boukouvalas, J. Kittler, R. Marik, and M. Petrou, "Automatic color grading of ceramic tiles using machine vision", IEEE Transactions on Industrial Electronics, Vol. 44, No. 1, 1997, pp. 132-135.

[9]. A. Jiaoyan, L. Di, and Z. Xuefeng, "Combination of wavelet analysis and color applied to automatic color grading of ceramic tiles", International Conference on Pattern Recognition, 2004, pp. 235-238.

[10]. I. Topalova, "Automated marble plate classification system based on different neural network input training sets and PLC implementation", International Journal of Advanced Research in Artificial Intelligence, Vol. 1, No. 2, 2012, pp. 50-56.

[11]. J.D. Luis-Delgado, J. Martinez-Alajarin, and L.M. Tomas-Balibrea, "Classification of marble surfaces using wavelets", Electronics Letters, Vol. 39, No. 9, 2003, pp. 714-715.

[12]. X. Xu, H. Liu, Y. Li, and Y. Zhou, "Image deblurring with blur kernel estimation in RGB channels", International Conference on Digital Signal Processing, 2016, pp. 681-684.

[13]. Y. Feng, G. Ren, K. He, Y. Liu, and L. Li, "RGB color channel variation based segmentation of crop leaf lesion", IEEE $10^{\text {th }}$ Conference on Industrial Electronics and Applications, 2015, pp. 592-596.

[14]. S. Kido, Y. Hirano, and N. Hashimoto, "Detection and classification of lung abnormalities by use of convolutional neural network (CNN) and regions with CNN features (R-CNN)", International Workshop on Advanced Image Technology, 2018, pp. 1-4.

[15]. I. Vornicu, and L. Goras, "On the design of a class of CNN's for ECG classification", European Conference on Circuit Theory and Design, 2011, pp. 150-153.

[16]. VxC TSG Database: http://miron.disca.upv.es/vision/vxctsg/ 